\title{
Real-Time 3D Body Scanning
}

\author{
Minoru NIIMURA ${ }^{a}$, Matthew W. BELLIS ${ }^{a}$, Daniel L. LAU ${ }^{b}$ \\ ${ }^{a}$ SEIKOWAVE, Kawasaki, Japan; \\ ${ }^{\mathrm{b}}$ University of Kentucky, Lexington (KY), USA
}

\begin{abstract}
Phase measurement profilometry (PMP) is a well-known technique for making 3D measurements. The technique involves the projection of patterns with a sinusoidally varying spatial intensity. This approach has been used extensively to make highly accurate measurements of static images. The use of structured light to make highly accurate measurements on human subjects is more difficult because of the inherent motion of the subject under test.

This document describes the principal of operation and the capability of SEIKOWAVE technology how it realizes the 3D full body scan and extends to the real-time 3D body scanning. This document also describes about the handheld microscopic 3D scanner developed by SEIKOWAVE.
\end{abstract}

\section{Principal of operation of SEIKOWAVE 3D body scanning system "JTS400"}

JTS400 Full Body Scanner is based on a configuration of four JTS100 towers, Figure1. Each JTS100 tower contains three LAMM 3D scanners (for example, the LAMM-01-MP01). The standard LAMM 3D scanner is configured with a $656 \times 492$ sensor. This sensor acquires approximately 300,000 3D measurement points; for a typical JTS400 configuration, the resolution is approximately $+/-2 \mathrm{~mm}$. The LAMM scanner with option G125 increases the standard resolution from 656 X 492 to 1292 X 964; with this option, the 3D scanner acquires approximately 1,200,000 3D measurement points per scan. A JTS400 configured with this option will typically have $+/-0.5 \mathrm{~mm}$ resolution.

The LAMM 3D scanner acquires 3D measurement points using patent-pending real-time structured light algorithms developed by SEIKOWAVE in cooperation with the University of Kentucky. These algorithms are based on phase measurement profilometry, or PMP. The main components of the LAMM 3D scanner are a camera, a pattern generator, and a projector. The pattern generator supplies specific patterns to the projector. The projector projects these patterns onto an object under test. The camera captures the object under test with the patterns projected across the surface of the object. The three dimensional shape of the surface of the object under test distorts the projected pattern. The projected PMP pattern is shown in equation (1).

$$
I_{n}^{p}\left(\mathrm{x}^{p}, y^{p}\right)=A^{p}+B^{p} \cos \left(2 \pi f y^{p}-\left(\frac{2 \pi n}{N}\right)\right)
$$

Where $\mathrm{f}$ is the spatial frequency of the projected pattern, $\mathrm{p}$ refers to the projected pixel and $A^{p}$ and $B^{p}$ are coefficients generally chosen to maximize the dynamic range of the projection system; $x$ and $y$ refer to the coordinate system of the projection system. PMP systems project a series of patterns. The total number of patterns is indicated by $N$. The intensity associated with a specific pattern is indicated by $n$.

The camera in the measurement system, which is positioned such that the camera, the projector, and the object under test form a triangle, captures images that can be described by equation (2).

$$
I_{n}^{c}\left(\mathrm{x}^{c}, y^{c}\right)=A^{c}\left(\mathrm{x}^{c}, y^{c}\right)+B^{c}\left(\mathrm{x}^{c}, y^{c}\right) \cos \left(\phi^{w}\left(\mathrm{x}^{c}, y^{c}\right)-\left(\frac{2 \pi n}{N}\right)\right)
$$

In equation (2), the term $\phi^{w}$ is referred to as the wrapped phase term and, when properly unwrapped and combined with the system calibration, is used to determine the depth or z-axis position of the specific pixel under examination (the $x$-axis and $y$-axis positions are determined by the camera pixel coordinates and the system calibration).

The phase term in (2) can be calculated as shown in equation (3).

$$
\phi^{w}\left(\mathrm{x}^{c}, y^{c}\right)=\arctan \left[\frac{\sum_{n=0}^{N-1} I_{n}^{c}\left(\mathrm{x}^{c}, y^{c}\right) \cos \left(\frac{2 \pi n}{N)}\right)}{\sum_{n=0}^{N-1} I_{n}^{c}\left(\mathrm{x}^{c}, y^{c}\right) \sin \left(\frac{2 \pi n}{N}\right)}\right]
$$


Typically, the calculation of the arctangent function in (3) has caused PMP measurement techniques to be slow. SEIKOWAVE has implemented an LUT (lookup table) based approach that simplifies the operations and significantly reduces the time to acquire and display 3D data. The values of the LUT are determined by the number of PMP patterns and the bit-depth of the camera system. In effect, all potential arctangent outcomes are, a priori, determined. This approach not only greatly increases the speed of the measurement but makes it possible to render the 3D measurement real-time on a display. This latter capability is extremely useful when making measurements of human subjects.

Equations (4) through (8) define the key parameters that are used in the reconstruction of 3D images and in determining the quality of the 3D data.

$$
\begin{aligned}
& A^{c}=\frac{1}{N} \sum_{n=0}^{N-1} I_{n}^{c}\left(\mathrm{x}^{c}, y^{c}\right) \\
& B^{c}{ }_{R}=\sum_{n=0}^{N-1} I_{n}^{c}\left(\mathrm{x}^{c}, y^{c}\right) \cos \left(\frac{2 \pi}{N}\right) \\
& B^{c}{ }_{I}=\sum_{n=0}^{N-1} I_{n}^{c}\left(\mathrm{x}^{c}, y^{c}\right) \sin \left(\frac{2 \pi}{N}\right) \\
& B^{c}=\left\|B^{c}{ }_{R}+j B^{c}{ }_{I}\right\|=\left\{B_{c_{R}}{ }^{2}+j B_{c_{I}}{ }^{2}\right\}^{\frac{1}{2}} \\
& \phi^{w}\left(\mathrm{x}^{c}, y^{c}\right)=\arctan \left\{\frac{B^{c} I}{B^{c}{ }_{R}}\right\}
\end{aligned}
$$

The $B^{c}$ coefficient is used as a quality measure of the pixel data. A measured point in which the $B^{c}$ value is strong indicates a point in which intensity values of successive projected patterns varies strongly: data from this pixel will likely result in a 3D position calculation with a high signal-to-noise. Weak $B^{c}$ values typically result from pixels in which the measured intensity of successive PMP patterns does not vary greatly. These pixels are often focused on points that are in shadow are have low reflectance. However, saturation of the camera pixel by the return signal can also result in a weak $B^{c}$ value. Pixels with weak $B^{c}$ values should be discarded.

In addition to using the $B^{c}$ coefficient for noise estimation, SEIKOWAVE has developed a method of using Fourier components to estimate motion. Ignoring for the moment system noise, for a static object, the wrapped phase $\phi^{w}\left(x^{c}, y^{c}\right)$ from equation (8) will be a constant. By treating the samples measured by the camera as a single period of a discrete-time signal, Lau [3] showed that information about the signal could be extracted from the Fourier coefficients of a discrete-time Fourier transform. Namely:

$$
\begin{aligned}
& A^{c}=\frac{1}{N} X[0] \\
& B^{c}=\frac{2}{N}\|X[1]\|
\end{aligned}
$$

Where $X[0]$ and $X[1]$ are the first two coefficients. Under ideal conditions, all of the signal should reside in the $X[0], X[1]$, and $X[N-1]$ terms: these are the principal frequencies of the system. The $X[2]$, $X[3], X[N-2]$ terms should all be zero. However, system noise will cause the coefficients of the principal frequencies to decline and those of the non-principal frequencies to increase. The second harmonic, the $\mathrm{X}$ [2] coefficient, is used to determine if the object was in motion during data acquisition.

Both noise thresholds and motion thresholds can be set in software, as illustrated in Figure 2.

Figure 3 shows Ac coefficient, Bc coefficient and phase image from a scan of a hand. 


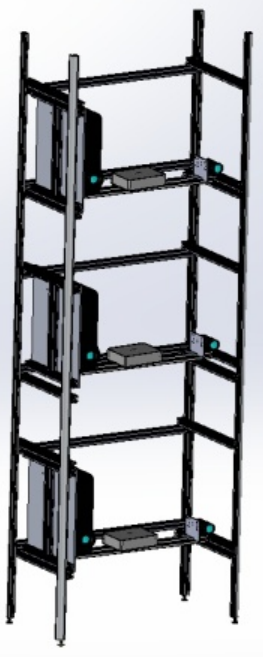

Fig. 1. JTS100 Tower.

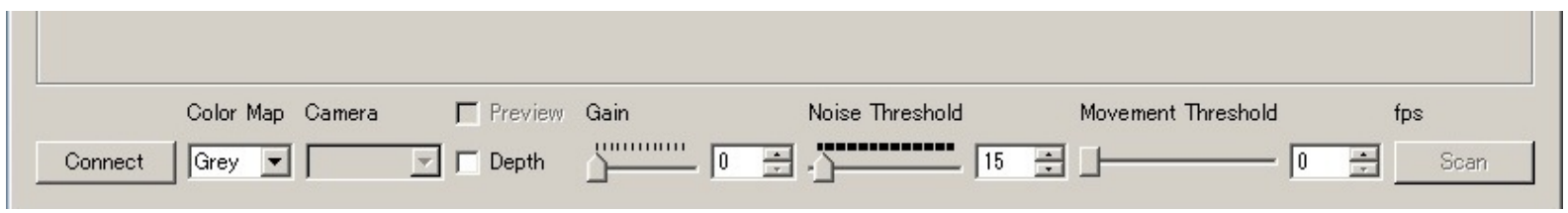

Fig. 2. JTS100 GUI.
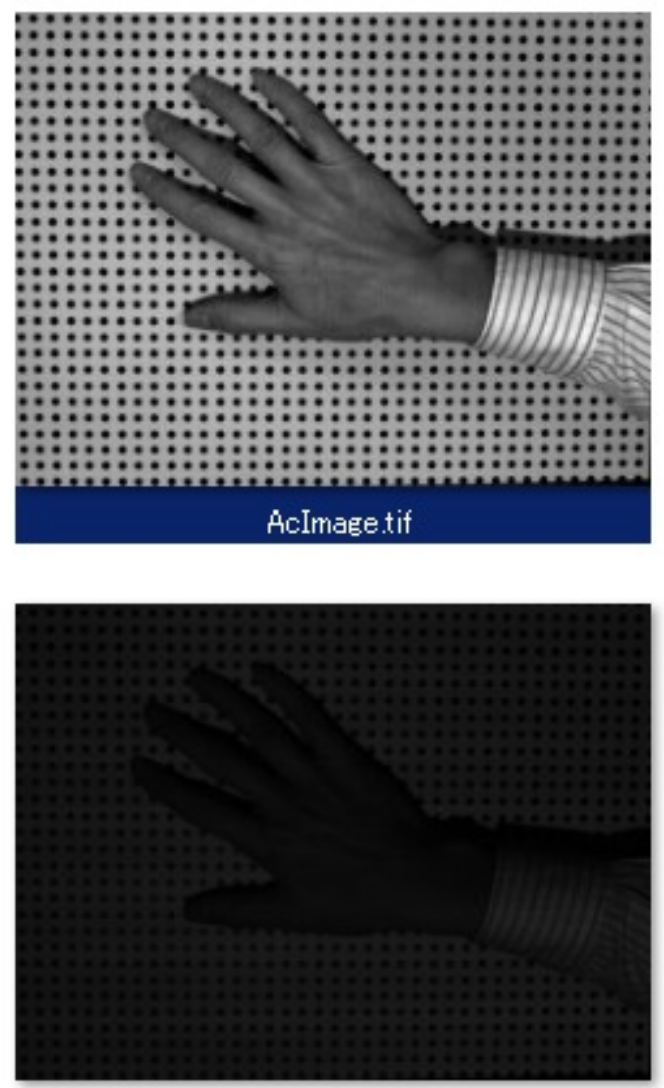

DcImage.tif

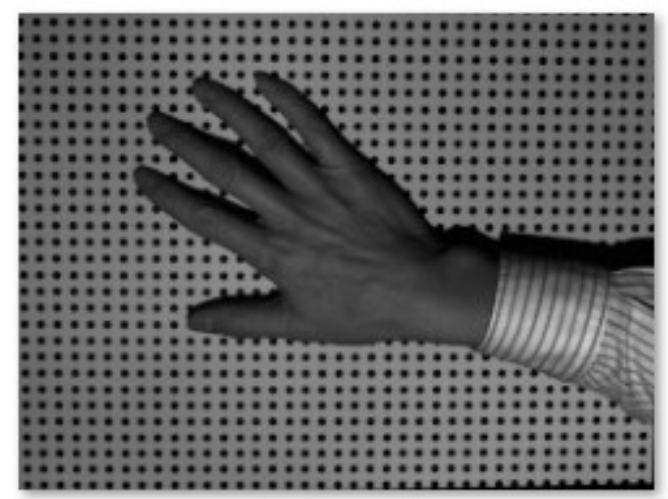

BcImage.tif

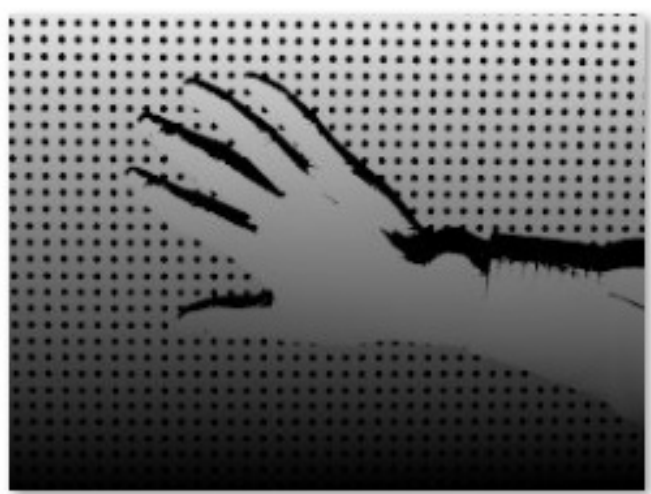

PhaseImage.tif

Fig. 3. $A c, B C$ and Phase Image of a $3 D$ scan. 


\section{Real-time point cloud generation}

\subsection{Capturing moving hand \& fingers}

JTS100 tower, Figure 1, utilizes SEIKOWAVE patent pending real-time structured light algorithm, however, the outcome of the scan is still static 3D image.

Figure 4 below shows how SEIKOWAVE algorithm generates "real-time" 3D image.

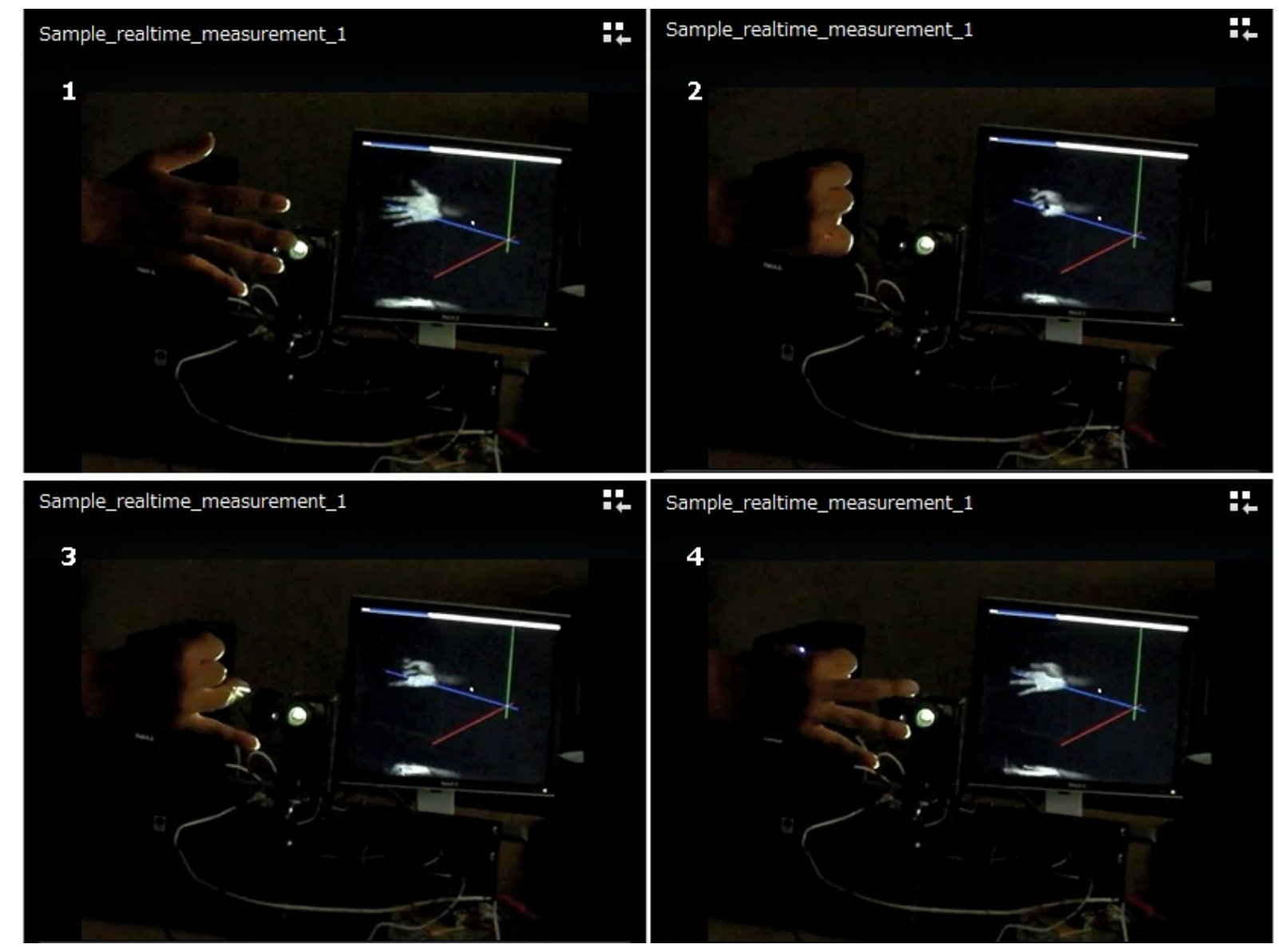

Fig. 4. Real-time 3D scanning.

These shots were captured from the real-time movie showing how the hand and fingers were captured into 3D point clouds. Frame 1 shows the initial shape of the hand. The white hand in the computer screen is the 3D point cloud of the hand. Frame 2, 3, and 4 shows different number of fingers and the computer screen follows the exact shape in real-time. These experiments are based on an "off-the-shelf" DLP projector and an industrial class image sensor. Both devices run at $60 \mathrm{~Hz}$ for this case. The rate of the point cloud generation is about 15 frames per second.

\subsection{Ultra fast 3D point cloud generation}

With SEIKOWAVE algorithm, 15 frames 3D point cloud per second is achieved with $60 \mathrm{~Hz}$ systems. As described in the previous chapter. SEIKOWAVE has been working with a camera vendor who has 1,000 fps or faster camera. The following figure, Figure 5, illustrates how the projected patterns and the camera capture work in synchronized manner. 

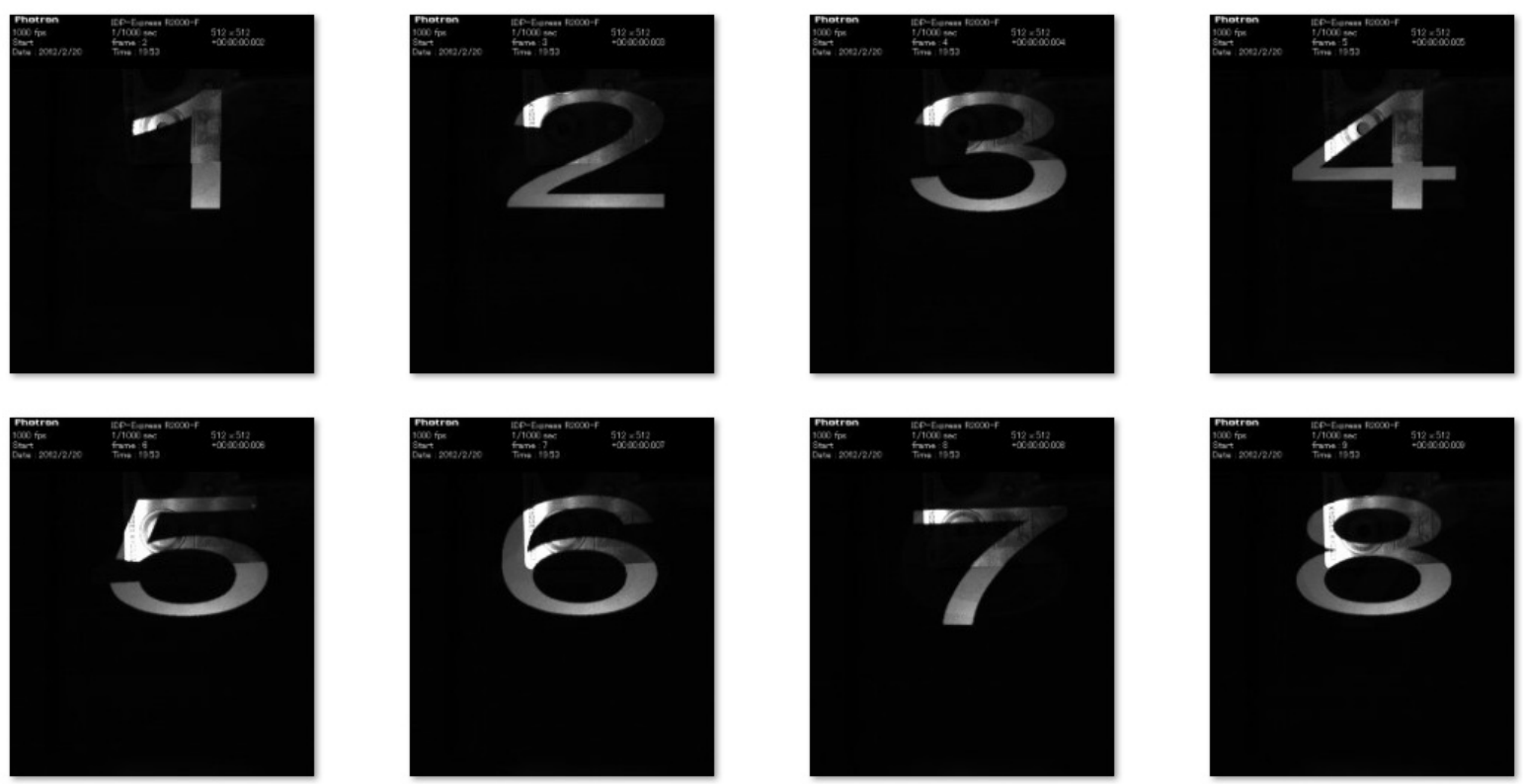

Fig. 5. Synchronization with a camera at 1,000fps.

The photos above were captured by a camera with exposure time 500us and the frame rate at 1,000 frames per second. There are 8 figures shown above meaning that the projector and the camera work at the exact same timing. With this experiment, 8 figures were used in order to clearly distinguish one frame and the next frame. When a PMP pattern is used, the reflection is also captured in an exact timing so that the point cloud generation will be possible with this rate at 1,000fps. We are also working even much faster rate at $10 \mathrm{KHz}$ to measure the road condition from the running vehicle.

\section{Application}

When a real-time 3D body scanning is achieved, we can think of a lot of applications including medical cases;

- Gait analysis

- $\quad$ Lung volume measurement or breath monitoring

- Gesture recognition or remote controller free command input

- Physical exercise

- $\quad$ Sports training (swing analysis; golf, tennis, baseball and so forth)

- Animal body measurement

- Etc.

There are many other, non-body related applications utilizing real-time measurement;

- Transient analysis (monitoring during heat cycle, monitoring of impact or crashes,,.,)

- In line measurement (products on a belt conveyer)

- Road condition analysis from a running car

- $\quad$ Etc.

\section{Products}

In addition to JTS400/100, SEIKOWAVE has been developing several different types of 3D scanners.

Figure 6 shows a real-time 3D shape monitoring system including a temperature chamber. It uses the ultra fast camera $(1,000 \mathrm{fps})$ to monitor board warping while high temperature is applied. 


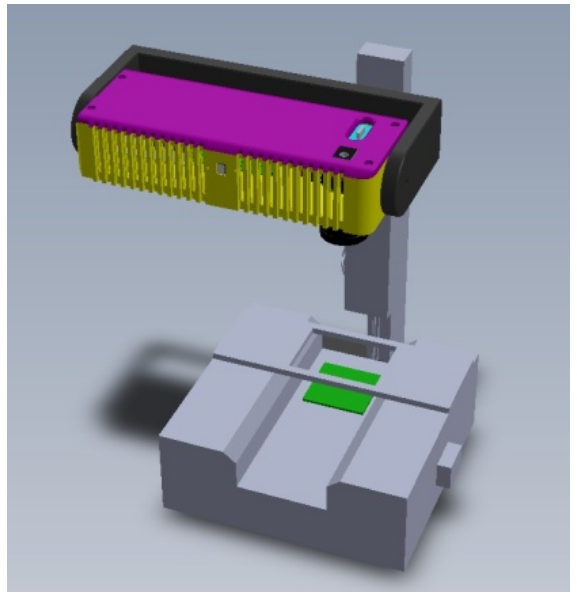

Fig. 6. Real-time measurement system.

Figure 7 shows the body scanning unit in an enclosure. Three units of this box consists a scanning tower JTS100.

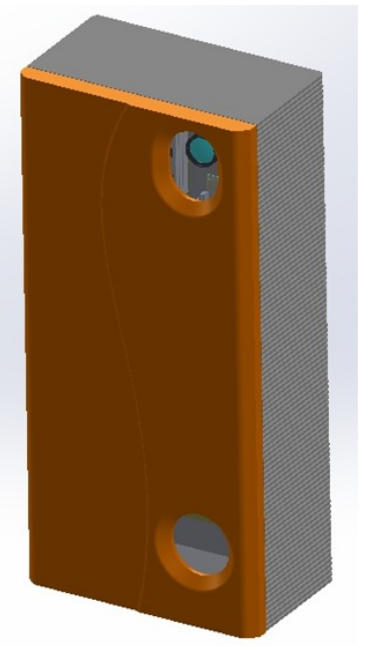

Fig. 7. JST100 in an enclosure.

Figure 8 shows a prototype model of a handy style 3D microscope. This implements a MEMS pattern generator developed by SEIKOWAVE. Images per every 8ms are captured and generate 3D point cloud from 8 contiguous frames. The resolution of the point cloud is $25 \mathrm{um}$.

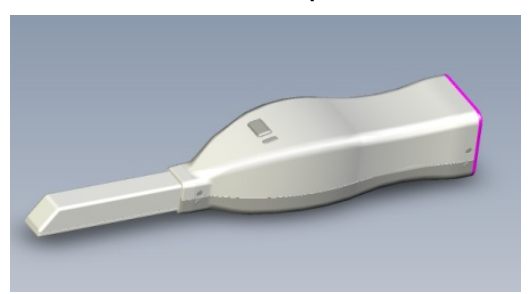

Fig. 8. Prototype model of a handy style 3D microscope.

\section{References}

1. SEIKOWAVE (2012), "Body scanner manual, Rev. 1.0",

2. Kai Liu, Yongchang Wang, Daniel L. Lau, Qi Hao, Laurence G. Hassebrook (2010): "Dual-frequency pattern scheme for high-speed 3-D shape measurement"

3. Matthew W. Bellis, Daniel L. Lau, "Measurement of human subjects using structured light" (2012) 